\title{
新型吡唑酰基硫腿类化合物的设计合成、杀菌活性与分子对接研究
}

\author{
孙娜波 ${ }^{a}$ 沈钟华 ${ }^{b}$ 翟志文 ${ }^{b}$ 韩 亮 ${ }^{b}$ \\ 翁建全 ${ }^{b}$ 谭成侠 ${ }^{b}$ 刘幸海 $*, b$ \\ ( ${ }^{a}$ 浙江树人大学生物与环境工程学院 杭州 310015)

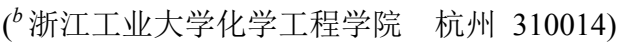

\begin{abstract}
摘要 以乙酰乙酸乙酯、原甲酸三乙酯、甲基肼等为原料，通过多步反应制备了一系列吡唑酰基硫艮类化合物，产物 结构均经过 ${ }^{1} \mathrm{H} N M R,{ }^{13} \mathrm{C}$ NMR 和 HRMS 确证. 对所有化合物进行了杀菌活性测试, 结果表明部分化合物对苹果轮纹病 具有较好的抑制效果，同时将高活性化合物 $N$-(2,6-二乙基苯基)-1,3-二甲基- $1 H$-吡唑-4-酰基硫嫝(6k)与琥珀酸脱氢酶进 行了对接. 对接结果表明, 化合物 $6 \mathbf{k}$ 能与琥珀酸脱氢酶形成稳定的复合物, 形成氢键和 $\pi(\sigma)-\pi$ 相互作用.
\end{abstract}

关键词 吡唑; 酰基硫脲; 合成; 杀菌活性; 分子对接

\section{Design, Synthesis, Fungicidal Activity and Docking Study of Acyl Thiourea Derivatives Containing Pyrazole Moiety}

\author{
Sun, Nabo ${ }^{a} \quad$ Shen, Zhonghua ${ }^{b} \quad$ Zhai, Zhiwen $^{b} \quad$ Han, Liang ${ }^{b}$ \\ Weng, Jianquan $^{b} \quad$ Tan, Chengxia $^{b} \quad$ Liu, Xinghai*,b \\ ( ${ }^{a}$ College of Biology and Environmental Engineering, Zhejiang Shuren University, Hangzhou 310015) \\ ( ${ }^{b}$ College of Chemical Engineering, Zhejiang University of Technology, Hangzhou 310014)
}

\begin{abstract}
A series of novel acyl thiourea derivatives containing pyrazole moiety were designed and synthesized from ethyl acetoacetate, triethyl orthoformate, methylhydrazine by multi-step reactions. Their structures were characterized by ${ }^{1} \mathrm{H}$ NMR, ${ }^{13} \mathrm{C}$ NMR and HRMS. The target compounds were evaluated for their fungicidal activity. The results indicated that some of the title compounds displayed certain fungicidal activities against Botryospuaeria berengeriana. The docking results indicated that the hydrogen bond and $\pi(\sigma)-\pi$ interaction formed between $N$-((2,6-diethylphenyl)carbamothioyl)-1,3-dimethyl-1H-pyrazole-4carboxamide (6k) and succinodehydrogenase.
\end{abstract}

Keywords pyrazole; acyl thiourea; synthesis; fungicidal activity; docking

琥珀酸脱氢酶抑制剂作用于病原菌线粒体呼吸电 子传递链上的琥珀酸脱氢酶或琥珀酸-泛醌还原酶, 是 被国际杀菌剂抗性行动委员会(FRAC)新划分出来的一 类作用机制和抗性机理相似的化合物 ${ }^{[1]}$. 自从 20 世纪 60 年代莠锈灵(carboxin)和氧化莠锈灵(oxycarboxin)问 世后, 靶向琥珀酸脱氢酶的杀菌剂已成为农业生产上的 一类非常重要的品种. 近年来, 一大批新颖结构的该类 杀菌剂被开发出来 ${ }^{[2]}$, 如联苯吡菌胺、氟唑环菌胺、氟 唑菌酰胺、吡唑菜菌胺、吡噻菌胺、呋吡菌胺、苯并烯 氟菌唑等. 该类杀菌剂分子结构变化比较大, 且近年来 发现的大多含吡唑环 ${ }^{[3 \sim 5]}$, 如杨光富课题组 ${ }^{[6,7]}$ 利用药效
团片段虚拟篎选(PFVS)方法创制出了新吡唑酰胺琥珀 酸脱氢酶抑制剂: 氟苯醚酰胺. 本课题组也设计合成了 多个系列的长链吡唑酰胺类化合物, 发现该类化合物具 有很好的杀线虫活性 ${ }^{[8 \sim 12]}$ 和杀菌活性 ${ }^{[13,14]}$. 另一方面, 硫脲类化合物因具有除草 ${ }^{[15]}$ 、杀虫 ${ }^{[16]}$ 、杀菌 ${ }^{[17]} 、$ 灭蚊 ${ }^{[18]}$ 、 抗癌 ${ }^{[19]}$ 等多样性的生物活性而受到广泛关注. 本课题 组在商品化琥珀酸脱氢酶抑制剂和前期工作 ${ }^{[20 ~ 25]}$ 的基 础上, 将脲结构变换成硫脲结构 ${ }^{[26]}$, 合成了 11 个未见报 道的吡唑酰基脲类新化合物 $\mathbf{6 a} \sim \mathbf{6 k}$, 设计思路如图 1 所 示.

\footnotetext{
* Corresponding author. E-mail: xhliu@zjut.edu.cn

Received April 18, 2017; revised June 2, 2017; published online June 16, 2017.

Project supported by the Natural Science Foundation of Zhejiang Province (No. LY16C14007) and the National Natural Science Foundation of China (No. 31401691).

浙江省自然科学基金(No. LY16C14007)和国家自然科学基金(No. 31401691)资助项目.
} 


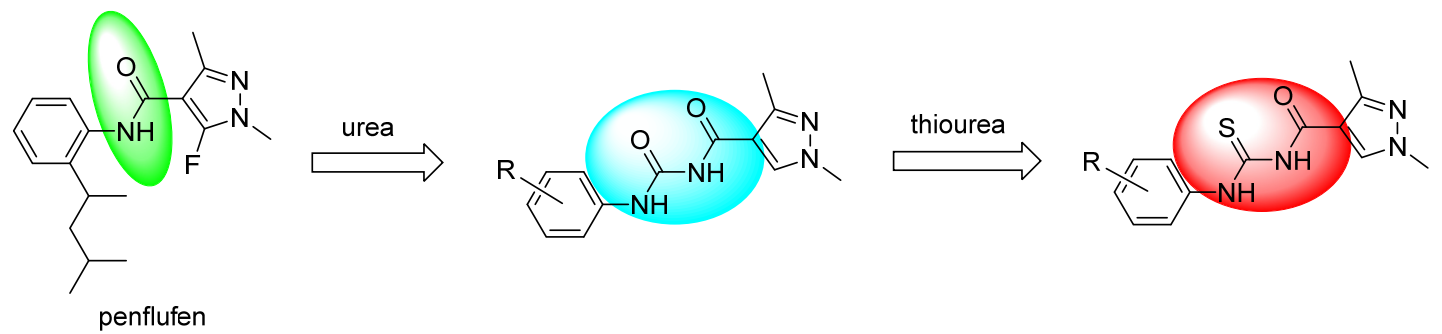

图 1 目标化合物的设计策略

Figure 1 Design strategy of title compounds

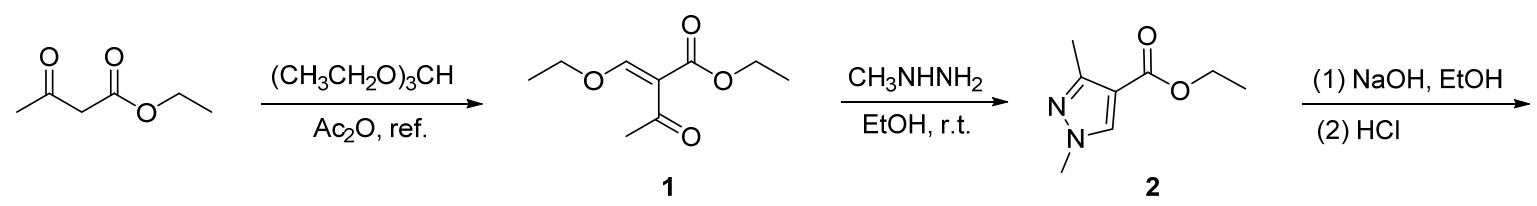

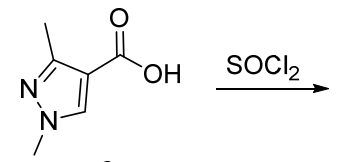

3

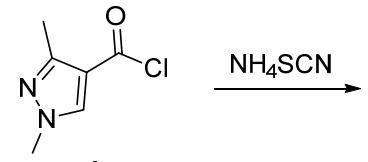

4

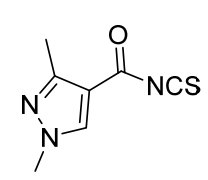

5

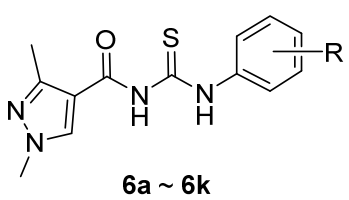

$6 a \sim 6 k$

6a: $R=2-F, 6 b R=3-F, 6 c: R=3,4-F_{2}, 6 \mathbf{d}: R=2,6-F_{2}, 6 e: R=2-O_{3} F_{3}, 6 f: R=2-C F_{3}, 6 g: R=2-C_{3}, 6 h: R=3,5-\left(C H_{3}\right)_{2}, 6 i: R=4-E t$, 6j: $R=2,3,4-F_{3}, 6 \mathbf{k}: R=2,6-E_{2}$

图式 1 吡唑酰基硫脲类化合物的合成路线

Scheme 1 Synthetic route of pyrazole acyl thiourea compounds

\section{1 结果与讨论}

\section{1 合成}

目标化合物的合成路线如 Scheme 1 所示. 以乙酰 乙酸乙酯与原甲酸三乙酯为原料, 经过缩合、环化两步 反应制得关键中间体 1-甲基-3-甲基- $1 H$-吡唑-4-甲酸乙 酯(2), 然后进行水解反应得到 1-甲基-3-甲基- $1 H$-吡 唑-4-甲酸(3). 再是与氯化试剂二氯亚砜反应得到吡唑 酰氯 4 , 未经纯化直接用于下一步反应. 在制备酰氯的 过程中, 二氯亚砜既是反应物又充当溶剂. 反应结束后, 需将多余的二氯亚砜除净, 否则对后续的酰基异硫氧酸 酯的制备有较大的影响; 同时通过加入 PEG-600, 可以 提高酰基异硫氰酸酯的转化率. 由于生成的酰基异硫氰 酸酯易发生聚合或者水解, 在反应制备得到吡唑酰基异 硫氰酸酯后, 及时与取代的胺反应, 产生氯化铵沉淀, 可以进行过滤除去. 最终得到的吡唑酰基硫脲纯度高, 且易于通过重结晶进一步纯化.

合成的化合物 $6 \mathrm{a} \sim 6 \mathrm{k}$ 的核磁共振谱、高分辨质谱 符合 Scheme 1 所示结构. 以高活性化合物 $\mathbf{6 k}$ 的波谱数 据为例进行说明, 化学位移 $\delta$ 在 9.01 (CONHCS) 和 11.91 $(\mathrm{ArNH})$ 处的单峰分别为酰基硫脲桥上两个 $\mathrm{N}-\mathrm{H}$ 的质 子信号峰, 后者的位移稍大一些是由于 $\mathrm{ArNH}$ 中的 $\mathrm{NH}$ 可以和羰基中的氧原子形成分子内氢键, 形成较稳定的
六元环状结构; $\delta 7.96$ 处的单峰为吡唑环上氢的质子信 号峰; $\delta 3.89$ 和 2.54 处的单峰分别为吡唑环上相连的 N甲基和 $\mathrm{C}$-甲基上氢的质子信号峰; 其余分别为苯环上 氢的质子信号峰和两个乙基的质子信号峰; 同时高分辨 质谱结果显示与分子量保持一致.

\section{2 生物活性}

番茄早疫病菌(Alternaria solani)、小麦赤霉病菌 (FusaHum graminearum)、马铃薯晚疫病菌(Phytophthora infestans)、辣椒疫霉病菌(Phytophthora capsici)、黄瓜灰 霉病菌 (Botrytis cinerea)、油菜菌核病菌 (Sclerotinia sclerotiorum)、水稻纹枯病菌(Thanatephorus cucumeris)、 花生褐斑病菌(Cercospora arachidicola)、苹果轮纹病菌 (Botryospuaeria berengeriana) 的杀菌活性列于表 1, 测 试浓度为 $50 \mu \mathrm{g} / \mathrm{mL}$. 由表 1 可知, 在 $50 \mu \mathrm{g} / \mathrm{mL}$ 的浓度下, 11 个新吡唑酰基硫脲化合物对供试菌种均表现出一定 的抑制活性. 但整体来看, 在 11 个新化合物中, 化合物 6k 表现出较好的杀菌活性, 尤其是对于苹果轮纹病的 抑制活性与对照药氟唑菌酰胺相当, 同时对花生褐斑病 (70.0\%)、黄瓜枯萎病 $(61.1 \%)$ 、水稻纹枯病 $(69.8 \%)$ 和番 茄早疫病(64.5\%)等也有中等的抑制活性. 此外, 化合物 6a, 6b, $6 \mathrm{~g}$ 和 6i 对苹果轮纹病也同样表现出了较好的杀 菌活性(73\% 85\%). 对番茄早疫病, 大部分化合物具 有中等的抑制活性. 如化合物 6a, 6f, 6g, 6h, 6i, 6j 和 6k 
对番茄早疫病的抑制活性达中等 $(42 \% \sim 65 \%)$, 逊于对 照药氟唑菌酰胺 $(88.9 \%)$. 对于油菜菌核病, 部分化合物 表现出中等强度的杀菌活性，如化合物 $6 \mathbf{a}(53.8 \%), \mathbf{6 b}$ $(53.8 \%), 6 \mathbf{c}(69.2 \%)$ 和 $6 \mathrm{~g}(57.7 \%)$. 对于黄瓜灰霉病，化 合物 6a $(61.0 \%)$ 和 $6 \mathbf{b}(61.0 \%)$ 的抑制活性与对照药氟唑 菌酰胺 $(63.6 \%)$ 相当, 化合物 6c (48.8\%)和 6g (56.1\%)也 表现出了中等强度的抑制活性. 对于小麦赤霉病、马铃 薯晚疫病、辣椒疫霉病, 对照药氟唑菌酰胺几乎无抑制 作用，而化合物 $6 \mathrm{a} \sim 6 \mathrm{e}$ 对辣椒疫霉病具有中等强度的 抑制活性(42\% 50\%), 其它两个菌种也几乎没有活性. 从表 2 可以看出化合物 $6 \mathbf{b}$ 和 $6 \mathbf{k}$ 对苹果轮纹病具有优异 的抑制活性, 对苹果轮纹病的 $\mathrm{EC}_{50}$ 为 10.41 和 9.28 $\mu \mathrm{g} / \mathrm{mL}$, 与对照药氟唑菌酰胺相比 $\left(\mathrm{EC}_{50}=7.93 \mu \mathrm{g} / \mathrm{mL}\right)$ 活 性相当. 从目标化合物结构和杀菌活性的关系可以看出 (表 1), 当苯环的靠近硫脲桥的附近有大体积的取代基 时, 对苹果轮纹病的活性优于其他化合物; 而当含氟的 取代基变换为三氟甲基时, 其活性降低, 说明电负性增 大不利于提高活性.

\section{3 分子对接}

从 PDB 库(http://www.rcsb.org)下载琥珀酸脱氢酶 与萎锈灵的复合晶体 (PDB:2FBW) 的 PDB 文件. 蛋白 结构的预处理在 Discovery Studio 2.5 软件中进行, 同时 对目标化合物 6k 进行结构优化, 采用 CDOCK 模块进行 分子对接, 输出 20 个对接结果, 选取打分最高的对接构 象进行分析. 本工作采用结合萎锈灵的琥珀酸脱氢酶复 合物晶体结构作为对象进行分子对接研究 ${ }^{[27,28]}$. 如图 2 所示, 目标化合物 $\mathbf{6 k}$ 与 SDH 的相互作用图. 其中, 目 标化合物 6k 脲桥上的氨基和吡唑环上的氮原子分别与 C 链上的 Ser39 和 His42 残基形成氢键作用, 同时吡唑 环与 B 链的 His 216 形成 $\pi-\pi$ 相互作用, 苯环与 $\mathrm{C}$ 链的 Ile40 形成 $\sigma-\pi$ 相互作用. 而商品化的先导化合物 penflufen 仅存在两个氢键, 分别是氟原子与 $\mathrm{C}$ 链上的
Ser39 和氧原子与 $\mathrm{B}$ 链的 $\operatorname{Trp} 173$ 残基形成氢键作用. 从 图 2 可见, 化合物 $\mathbf{6 k}$ 与 penflufen 与酶的结合位点邻近 $B$ 链和 C 链区域, 尤其是都与 Ser39 形成氢键. 同时, 化 合物 6k 引入硫脲桥之后, 化合物 $6 \mathbf{k}$ 与酶之间形成了更 为稳定的 $\pi-\pi$ 相互作用, 这可能是化合物 $6 \mathbf{k}$ 具有较高的 杀菌活性的原因.

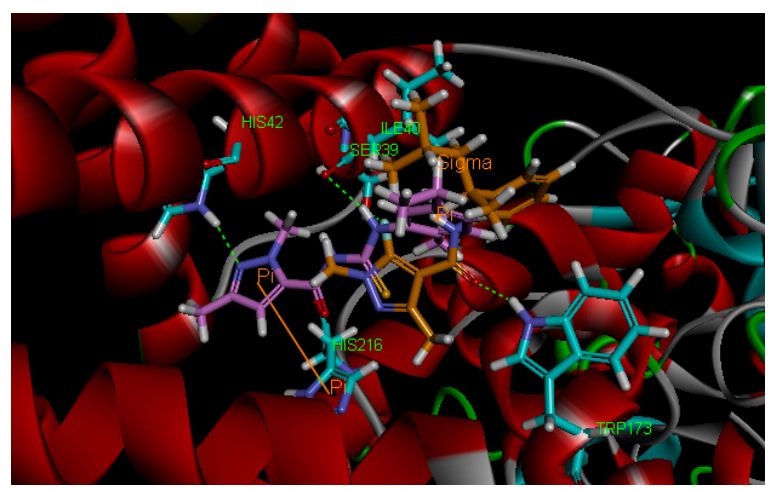

图 2 化合物 $6 \mathrm{k}$ 的分子对接图

Figure 2 Molecular docking of compound $6 \mathbf{k}$

\section{2 结论}

本文以乙酰乙酸乙酯为原料, 经多步反应合成出一 系列新型吡唑酰基硫脲类化合物，目标化合物经核磁共 振氢谱和高分辨质谱分析确定其结构. 以番茄早疫病菌 (Alternaria solani)、小麦赤霉病菌(Fusahum graminearum)、马铃薯晚疫病菌(Phytophthora infestans)、辣椒 疫霉病菌(Phytophthora capsici)、黄瓜灰霉病菌(Botrytis cinerea)、油菜菌核病菌(Sclerotinia sclerotiorum)、水稻 纹枯病菌(Thanatephorus cucumeris)、花生褐斑病菌 (Cercospora arachidicola)、苹果轮纹病菌(Botryospuaeria berengeriana) 分别进行了杀菌活性笁选. 结果显示大部 分化合物对苹果轮纹病菌具有较好的杀菌活性, 尤其是 化合物 6k 可以作为先导化合物进一步优化.

表 1 目标化合物的杀菌活性(抑制率/\%)

Table 1 Fungicidal activity of title compounds (inhibition rate/\%)

\begin{tabular}{|c|c|c|c|c|c|c|c|c|c|c|c|}
\hline Compd. & $\mathrm{R}$ & 番茄早疫 & 小麦赤霉 & 马铃薯晚疫 & 辣椒疫霉 & 油菜菌核 & 黄瓜灰霉 & 水稻纹枯 & 黄瓜枯萎 & 花生褐斑 & 苹果轮纹 \\
\hline $6 a$ & $2-\mathrm{F}$ & 38.7 & 27.3 & 18.9 & 47.6 & 53.8 & 61.0 & 11.6 & 11.1 & 30.0 & 76.9 \\
\hline $6 b$ & $3-\mathrm{F}$ & 48.4 & 12.1 & 24.3 & 47.6 & 53.8 & 61.0 & 12.8 & 16.7 & 50.0 & 84.6 \\
\hline $6 c$ & $3,4-\mathrm{F}_{2}$ & 38.7 & 12.1 & 21.6 & 42.9 & 69.2 & 48.8 & 12.8 & 11.1 & 20.0 & 65.4 \\
\hline 6d & $2,6-\mathrm{F}_{2}$ & 32.3 & 45.5 & 16.2 & 42.9 & 19.2 & 22.0 & 17.4 & 27.8 & 20.0 & 53.8 \\
\hline $6 e$ & $2-\mathrm{OCF}_{3}$ & 29.0 & 15.2 & 21.6 & 45.2 & 38.5 & 24.4 & 17.4 & 22.2 & 20.0 & 46.2 \\
\hline $6 f$ & $2-\mathrm{CF}_{3}$ & 41.9 & 21.2 & 13.5 & 42.9 & 19.2 & 24.4 & 16.3 & 38.9 & 50.0 & 65.4 \\
\hline $6 g$ & $2-\mathrm{CH}_{3}$ & 45.2 & 21.2 & 16.2 & 50.0 & 57.7 & 56.1 & 23.3 & 22.2 & 10.0 & 73.1 \\
\hline $6 h$ & $3,5-\left(\mathrm{CH}_{3}\right)_{2}$ & 45.2 & 15.2 & 16.2 & 31.0 & 15.4 & 12.2 & 16.3 & 44.4 & 40.0 & 61.5 \\
\hline $6 \mathbf{i}$ & $4-E t$ & 48.4 & 36.4 & 18.9 & 38.1 & 26.9 & 29.3 & 11.6 & 33.3 & 30.0 & 73.1 \\
\hline $6 \mathbf{j}$ & $2,3,4-\mathrm{F}_{3}$ & 48.4 & 21.2 & 13.5 & 38.1 & 38.5 & 24.4 & 34.9 & 11.1 & 10.0 & 69.2 \\
\hline $6 k$ & 2,6-Et ${ }_{2}$ & 64.5 & 30.3 & 29.7 & 38.1 & 38.5 & 22.0 & 69.8 & 61.1 & 70.0 & 96.2 \\
\hline 氟唑菌酰胺 & & 88.9 & 30.3 & 29.7 & 38.1 & 96.4 & 63.6 & 88.4 & 44.4 & 100 & 84.6 \\
\hline
\end{tabular}


表 2 化合物 $6 \mathbf{b}$ 和 $6 \mathbf{k}$ 对苹果轮纹病的 $\mathrm{EC}_{50}$ 值 Table $2 \quad \mathrm{EC}_{50}$ of compounds $7 \mathbf{b}$ and $7 \mathbf{k}$

\begin{tabular}{cccc}
\hline Compd. & $y=a+b x$ & $r$ & $\mathrm{EC}_{50} /\left(\mu \mathrm{g} \cdot \mathrm{mL}^{-1}\right)$ \\
\hline $\mathbf{6 b}$ & $y=1.6661 x+3.2750$ & 0.9937 & 10.41 \\
$\mathbf{6 k}$ & $y=2.2238 x+2.8481$ & 0.9936 & 9.28 \\
氟唑菌酰胺 & $y=1.1363 x+3.9777$ & 0.9825 & 7.93 \\
\hline
\end{tabular}

\section{3 实验部分}

\section{1 仪器与试剂}

北京泰克仪器有限公司 X-4 数字显示显微熔点仪 温度计未校正; BRUKER $400 \mathrm{MHz}$ 和 $500 \mathrm{MHz}$ 核磁共 振仪 ( $\mathrm{CDCl}_{3}$ 为溶剂, TMS 为内标); 安捷伦 LC-QTOFMS 高分辨质谱仪. 试剂均采用分析纯试剂. 未经处理, 直接使用.

\section{2 实验方法}

关键中间体 1, 2, 3, 4 参考文献方法 ${ }^{[26,13]}$ 合成.

\subsection{1 目标化合物 $6 \mathrm{a} \sim 6 \mathrm{k}$ 的合成}

将吡唑酰氯化合物 $4(1.58 \mathrm{~g}, 10 \mathrm{mmol})$ 加入到无水 丙酮 $(20 \mathrm{~mL})$ 中, 加入硫氰酸铵 $(0.99 \mathrm{~g}, 13 \mathrm{mmol})$, 反应 体系即刻有沉淀生成并变成红色, 室温搅拌 $1 \mathrm{~h}$ 后反应 体系变为黄色, 继续反应 $1 \mathrm{~h}$, 反应停止, 抽滤除去固体 杂质, 减压蒸馏得淡黄色固体吡唑酰基异硫氧酸酯 $\mathbf{5}$, 无需纯化直接用于下一步反应.

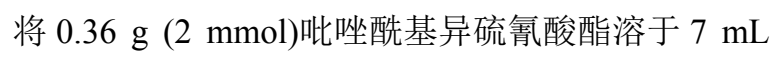
的 $\mathrm{CH}_{2} \mathrm{Cl}_{2}$ 中, 滴入 $2 \mathrm{mmol}$ 的取代苯胺 (对于固体的取代 苯胺, 将其溶于 $\mathrm{CH}_{2} \mathrm{Cl}_{2}$ 后再滴入), 加完后室温搅拌 12 $\mathrm{h}$, 生成固体, 抽滤, $\mathrm{CH}_{2} \mathrm{Cl}_{2}$ 洗涤, 干燥, 得系列化合物 $\mathbf{6 a} \sim 6 \mathbf{k}$.

$N$-(2-氟苯基)-1,3-二甲基- $1 H$ - 吡唑-4-酰基硫脲 (6a): 褐色固体, 产率 $60 \%$. m.p. $144 \sim 146{ }^{\circ} \mathrm{C} ;{ }^{1} \mathrm{H}$ NMR $\left(400 \mathrm{MHz}, \mathrm{CDCl}_{3}\right) \delta: 2.56\left(\mathrm{~s}, 3 \mathrm{H}, \mathrm{CH}_{3}\right), 3.92\left(\mathrm{~s}, 3 \mathrm{H}, \mathrm{CH}_{3}\right)$, $7.17 \sim 7.26$ (m, 4H, Ph), 7.96 (s, 1H, CH), 8.33 (t, $J=7.2$ $\mathrm{Hz}, 1 \mathrm{H}, \mathrm{Ph}), 8.93(\mathrm{~s}, 1 \mathrm{H}, \mathrm{NH}), 12.67(\mathrm{~s}, 1 \mathrm{H}, \mathrm{NH}),{ }^{13} \mathrm{C}$ NMR (125 MHz, $\left.\mathrm{CDCl}_{3}\right) \delta: 13.53,38.37,111.48,115.56$, $115.71,124.19,127.05,128.01,134.74,135.24,149.55$, $150.98,154.35,156.31,164.14,164.46,180.55$; HRMS calcd for $\mathrm{C}_{13} \mathrm{H}_{14} \mathrm{FN}_{4} \mathrm{OS}[\mathrm{M}+\mathrm{H}]^{+}$293.0867, found 293.0860.

$N$-(3-氟苯基)-1,3-二甲基- $1 H$-吡唑-4-酰基硫脲 $(\mathbf{6} \mathbf{b}$ ): 褐色固体, 产率 57.5\%. m.p. 136 138 ${ }^{\circ} \mathrm{C} ;{ }^{1} \mathrm{H}$ NMR (400 $\left.\mathrm{MHz}, \mathrm{CDCl}_{3}\right) \delta: 2.55\left(\mathrm{~s}, 3 \mathrm{H}, \mathrm{CH}_{3}\right), 3.93\left(\mathrm{~s}, 3 \mathrm{H}, \mathrm{CH}_{3}\right)$, 6.99 7.01 (m, 1H, Ph), 7.06 7.08 (m, 1H, Ph), $7.39(\mathrm{t}$, $J=2.0 \mathrm{~Hz}, 1 \mathrm{H}, \mathrm{Ph}), 7.73$ (d, $J=10.0 \mathrm{~Hz}, 1 \mathrm{H}, \mathrm{Ph}), 7.93$ (s, $1 \mathrm{H}, \mathrm{CH}), 8.79$ (s, 1H, NH), 12.73 (s, 1H, NH); ${ }^{13} \mathrm{C} \mathrm{NMR}$ $\left(125 \mathrm{MHz}, \mathrm{CDCl}_{3}\right) \delta: 13.51,38.67,108.99,109.20$,
$110.53,111.04,111.24,111.54,112.68,112.85,120.24$, $130.15,130.22,134.69,135.23,134.68,135.23,139.60$, $149.53,150.91,160.66,162.59,163.99,164.44,179.51$; HRMS calcd for $\mathrm{C}_{13} \mathrm{H}_{14} \mathrm{FN}_{4} \mathrm{OS}[\mathrm{M}+\mathrm{H}]^{+}$293.0867, found 293.0891.

$N$-((3,4-二氟苯基)-1,3-二甲基- $1 H$-吡唑-4-酰基硫艮 (6c): 褐色固体, 产率 $60.7 \%$. m.p. $155 \sim 157{ }^{\circ} \mathrm{C} ;{ }^{1} \mathrm{H}$ NMR (400 MHz, $\left.\mathrm{CDCl}_{3}\right) \delta: 2.54$ (s, $\left.3 \mathrm{H}, \mathrm{CH}_{3}\right), 3.92$ (s, $3 \mathrm{H}$, $\left.\mathrm{CH}_{3}\right), 7.14 \sim 7.22(\mathrm{~m}, 2 \mathrm{H}, \mathrm{Ph}), 7.78 \sim 7.82(\mathrm{~m}, 1 \mathrm{H}, \mathrm{Ph})$, $7.94(\mathrm{~s}, 1 \mathrm{H}, \mathrm{CH}), 8.86(\mathrm{~s}, 1 \mathrm{H}, \mathrm{NH}), 12.64(\mathrm{~s}, 1 \mathrm{H}, \mathrm{NH}) ;{ }^{13} \mathrm{C}$ NMR (125 MHz, $\left.\mathrm{CDCl}_{3}\right) \delta: 13.50,38.35,111.52,112.20$, $112.37,114.14,114.30,117.07,117.22,121.77,134.69$, 135.22, 149.52, 150.91, 163.90, 164.43, 180.04; HRMS calcd for $\mathrm{C}_{13} \mathrm{H}_{13} \mathrm{~F}_{2} \mathrm{~N}_{4} \mathrm{OS}[\mathrm{M}+\mathrm{H}]^{+}$311.0773, found 311.0768 .

$N$-(2,6-二氟苯基)-1,3-二甲基- $1 H$-吡唑-4-酰基硫豚 (6d): 淡黄色固体, 产率 46.7\%. m.p. 163 164 ${ }^{\circ} \mathrm{C} ;{ }^{1} \mathrm{H}$ NMR (400 MHz, $\left.\mathrm{CDCl}_{3}\right) \delta: 2.56\left(\mathrm{~s}, 3 \mathrm{H}, \mathrm{CH}_{3}\right), 3.93(\mathrm{~s}, 3 \mathrm{H}$, $\left.\mathrm{CH}_{3}\right), 7.04(\mathrm{t}, J=8.4 \mathrm{~Hz}, 2 \mathrm{H}, \mathrm{Ph}), 7.33 \sim 7.40(\mathrm{~m}, 1 \mathrm{H}, \mathrm{Ph})$, 7.91 (s, 1H, CH), 8.96 (s, 1H, NH), 11.83 (s, 1H, NH); ${ }^{13} \mathrm{C}$ NMR $\left(125 \mathrm{MHz}, \mathrm{CDCl}_{3}\right) \delta: 13.52,38.79,111.46,111.74$, $111.93,116.11,129.28,129.36,129.44,134.75,151.03$, 156.99, 157.02, 158.97, 159.01, 163.70, 182.47; HRMS calcd for $\mathrm{C}_{13} \mathrm{H}_{13} \mathrm{~F}_{2} \mathrm{~N}_{4} \mathrm{OS}[\mathrm{M}+\mathrm{H}]^{+}$311.0773; found 311.0771 .

$N$-(2-三氟甲氧基苯基)-1,3-二甲基- $1 H$-吡唑-4-酰基 硫嫝(6e): 淡黄色固体, 产率 50.6\%. m.p. $111 \sim 113{ }^{\circ} \mathrm{C}$; ${ }^{1} \mathrm{H}$ NMR $\left(400 \mathrm{MHz}, \mathrm{CDCl}_{3}\right) \delta: 2.57\left(\mathrm{~s}, 3 \mathrm{H}, \mathrm{CH}_{3}\right), 3.93$ (s, $\left.3 \mathrm{H}, \mathrm{CH}_{3}\right), 7.31 \sim 7.33(\mathrm{~m}, 1 \mathrm{H}, \mathrm{Ph}), 7.37 \sim 7.41(\mathrm{~m}, 2 \mathrm{H}$, $\mathrm{Ph}), 7.94$ (s, 1H, CH), 8.57 (d, J=8.4 Hz, 1H, Ph), 8.81 (s, $1 \mathrm{H}, \mathrm{NH}), 12.90(\mathrm{~s}, 1 \mathrm{H}, \mathrm{NH}) ;{ }^{13} \mathrm{C} \mathrm{NMR}\left(125 \mathrm{MHz}, \mathrm{CDCl}_{3}\right)$ $\delta: 13.45,38.79,111.40,121.48,127.20,127.41,127.65$, $131.02,134.80,135.24,141.22,150.98,164.36,180.47$; HRMS (ESI) calcd for $\mathrm{C}_{14} \mathrm{H}_{14} \mathrm{~F}_{3} \mathrm{~N}_{4} \mathrm{O}_{2} \mathrm{~S} \quad[\mathrm{M}+\mathrm{H}]$ 359.0784, found 359.0792.

$\mathrm{N}$-(2-三氟甲基苯基)-1,3-二甲基- $1 H$-吡唑-4-酰基硫 脉(6f): 淡黄色固体, 产率 55.3\%. m.p. $147 \sim 148{ }^{\circ} \mathrm{C} ;{ }^{1} \mathrm{H}$ NMR (400 MHz, $\left.\mathrm{CDCl}_{3}\right) \delta: 2.56\left(\mathrm{~s}, 3 \mathrm{H}, \mathrm{CH}_{3}\right), 3.92(\mathrm{~s}, 3 \mathrm{H}$, $\left.\mathrm{CH}_{3}\right), 7.46(\mathrm{t}, J=7.6 \mathrm{~Hz}, 1 \mathrm{H}, \mathrm{Ph}), 7.65(\mathrm{t}, J=8.0 \mathrm{~Hz}, 1 \mathrm{H}$, $\mathrm{Ph}), 7.75$ (t, $J=8.0 \mathrm{~Hz}, 1 \mathrm{H}, \mathrm{Ph}), 7.92$ (t, $J=8.4 \mathrm{~Hz}, 1 \mathrm{H}$, $\mathrm{Ph}), 7.96$ (s, 1H, CH), 8.98 (s, 1H, NH), 12.55 (s, 1H, NH); ${ }^{13} \mathrm{C}$ NMR $\left(125 \mathrm{MHz}, \mathrm{CDCl}_{3}\right) \delta$ : 13.51, 38.37, 111.40, $126.09,126.13,127.63,130.96,132.74,134.82,135.95$, $150.99,164.39,181.98$; HRMS (ESI) calcd for $\mathrm{C}_{14} \mathrm{H}_{14} \mathrm{~F}_{3}$ $\mathrm{N}_{4} \mathrm{OS}[\mathrm{M}+\mathrm{H}]^{+}$343.0835, found 343.0827 . 
$\mathrm{N}$-(2-氯苯基)-1,3-二甲基- $1 \mathrm{H}$-吡唑-4-酰基硫脲 $(\mathbf{6 g}$ ): 淡黄色固体, 产率 56.3\%. m.p. $175 \sim 176{ }^{\circ} \mathrm{C} ;{ }^{1} \mathrm{H}$ NMR $\left(400 \mathrm{MHz}, \mathrm{CDCl}_{3}\right) \delta: 2.57\left(\mathrm{~s}, 3 \mathrm{H}, \mathrm{CH}_{3}\right), 3.93\left(\mathrm{~s}, 3 \mathrm{H}, \mathrm{CH}_{3}\right)$, $7.25 \sim 7.27(\mathrm{~m}, 1 \mathrm{H}, \mathrm{Ph}), 7.37(\mathrm{~d}, J=8.0 \mathrm{~Hz}, 1 \mathrm{H}, \mathrm{Ph})$, $7.48 \sim 7.51(\mathrm{~m}, 1 \mathrm{H}, \mathrm{Ph}), 7.94(\mathrm{~s}, 1 \mathrm{H}, \mathrm{CH}), 8.31 \sim 8.33(\mathrm{~m}$, $1 \mathrm{H}, \mathrm{Ph}), 8.85$ (s, 1H, NH), 12.69 (s, 1H, NH); ${ }^{13} \mathrm{C}$ NMR $\left(125 \mathrm{MHz}, \mathrm{CDCl}_{3}\right) \delta: 13.54,38.79,111.48,111.57,127.20$, $127.23,128.07,128.22,128.35,129.18,129.44,134.79$, 135.24, 135.41, 150.96, 164.13, 164.46, 180.62; HRMS (ESI) calcd for $\mathrm{C}_{13} \mathrm{H}_{14} \mathrm{ClN}_{4} \mathrm{OS}[\mathrm{M}+\mathrm{H}]^{+}$309.0571, found 309.0564 .

$N$-(3,5-二甲基苯基)-1,3-二甲基- $1 \mathrm{H}$-吡唑-4-酰基硫 嫝(6h): 淡黄色固体, 产率 64.7\%. m.p. 142 143 ${ }^{\circ} \mathrm{C} ;{ }^{1} \mathrm{H}$ NMR (400 MHz, $\left.\mathrm{CDCl}_{3}\right) \delta: 2.36\left(\mathrm{~s}, 3 \mathrm{H}, \mathrm{CH}_{3}\right), 2.55(\mathrm{~s}, 3 \mathrm{H}$, $\mathrm{CH}_{3}$ ), 3.92 (s, 3H, $\left.\mathrm{CH}_{3}\right), 6.86$ (s, 1H, $\left.\mathrm{Ph}\right), 7.31$ (s, 2H, Ph), 7.92 (s, $1 \mathrm{H}, \mathrm{CH}), 8.77$ (s, $1 \mathrm{H}, \mathrm{NH}), 12.48(\mathrm{~s}, 1 \mathrm{H}, \mathrm{NH}),{ }^{13} \mathrm{C}$ NMR (125 MHz, $\left.\mathrm{CDCl}_{3}\right) \delta: 13.53,20.85,38.38,111.63$, $120.82,121.61,126.08,127.59,134.62,135.26,137.82$, $149.55,150.86,164.13,164.47,179.06$; HRMS (ESI) calcd for $\mathrm{C}_{15} \mathrm{H}_{19} \mathrm{~N}_{4} \mathrm{OS}[\mathrm{M}+\mathrm{H}]^{+}$303.1274, found 303.1276.

$\mathrm{N}$-(4-乙基苯基)-1,3-二甲基- $1 \mathrm{H}$-吡唑-4-酰基硫脲 (6i): 褐色固体, 产率 55.2\%. m.p. 89 90 ${ }^{\circ} \mathrm{C} ;{ }^{1} \mathrm{H}$ NMR $\left(500 \mathrm{MHz}, \mathrm{DCCl}_{3}\right) \delta: 1.26\left(\mathrm{t}, J=4.0 \mathrm{~Hz}, 3 \mathrm{H}, \mathrm{CH}_{3}\right), 2.54$ (s, $\left.3 \mathrm{H}, \mathrm{CH}_{3}\right), 2.66 \sim 2.70\left(\mathrm{~m}, 2 \mathrm{H}, \mathrm{CH}_{2}\right), 3.91\left(\mathrm{~s}, 3 \mathrm{H}, \mathrm{CH}_{3}\right)$, 7.25 (d, J=8.5 Hz, 2H, Ph), 7.94 (s, 1H, CH), 8.86 (s, $1 \mathrm{H}$, $\mathrm{NH}), 12.50$ (s, $1 \mathrm{H}, \mathrm{NH}) ;{ }^{13} \mathrm{C}$ NMR (125 MHz, $\left.\mathrm{CDCl}_{3}\right) \delta$ : 13.52, 15.50, 27.69, 38.38, 111.65, 123.31, 124.25, 127.85, $127.95,134.61,135.25,135.65,141.76,149.76,150.83$, 164.05, 164.46, 179.30; HRMS calcd for $\mathrm{C}_{15} \mathrm{H}_{19} \mathrm{~N}_{4} \mathrm{OS}$ $[\mathrm{M}+\mathrm{H}]^{+}$303.1274, found 303.1276 .

$N$-(2,3,4-三氟苯基)-1,3-二甲基- $1 H$-吡唑-4-酰基硫 脲(6j): 褐色固体, 产率 $42.2 \%$. m.p. 171 172 ${ }^{\circ} \mathrm{C} ;{ }^{1} \mathrm{H}$ NMR (400 MHz, $\left.\mathrm{CDCl}_{3}\right) \delta: 2.55\left(\mathrm{~s}, 3 \mathrm{H}, \mathrm{CH}_{3}\right), 3.92(\mathrm{~s}, 3 \mathrm{H}$, $\left.\mathrm{CH}_{3}\right), 7.00 \sim 7.06(\mathrm{~m}, 1 \mathrm{H}, \mathrm{Ph}), 7.86 \sim 7.90(\mathrm{~m}, 1 \mathrm{H}, \mathrm{Ph})$, $7.91(\mathrm{~s}, 1 \mathrm{H}, \mathrm{CH}), 8.87$ (s, $1 \mathrm{H}, \mathrm{NH}), 12.52(\mathrm{~s}, 1 \mathrm{H}, \mathrm{NH}) ;{ }^{13} \mathrm{C}$ NMR (125 MHz, $\left.\mathrm{CDCl}_{3}\right) \delta: 13.52,38.79,111.42,111.61$, $111.72,123.01,123.07,124.35,124.43,134.76,135.24$, 146.64, 147.84, 151.04, 163.90, 181.63; HRMS calcd for $\mathrm{C}_{13} \mathrm{H}_{12} \mathrm{~F}_{3} \mathrm{~N}_{4} \mathrm{OS}[\mathrm{M}+\mathrm{H}]^{+}$329.0678, found 329.0672.

$\mathrm{N}$-(2,6-二乙基苯基)-1,3-二甲基- $1 \mathrm{H}$-吡唑-4-酰基硫 脲(6k): 淡黄色固体, 产率 50.5\%. m.p. 111 112 ${ }^{\circ} \mathrm{C} ;{ }^{1} \mathrm{H}$ $\operatorname{NMR}\left(400 \mathrm{MHz}, \mathrm{CDCl}_{3}\right) \delta: 1.26\left(\mathrm{t}, J=7.5 \mathrm{~Hz}, 6 \mathrm{H}, 2 \mathrm{CH}_{3}\right)$, $2.54\left(\mathrm{~s}, 3 \mathrm{H}, \mathrm{CH}_{3}\right), 2.66 \sim 2.69\left(\mathrm{~m}, 4 \mathrm{H}, 2 \mathrm{CH}_{2}\right), 3.89(\mathrm{~s}, 3 \mathrm{H}$, $\left.\mathrm{CH}_{3}\right), 7.19(\mathrm{~d}, J=8.0 \mathrm{~Hz}, 2 \mathrm{H}, \mathrm{Ph}), 7.32(\mathrm{t}, J=7.5 \mathrm{~Hz}, 1 \mathrm{H}$,
Ph), 7.96 (s, 1H, CH), 9.01 (s, 1H, NH), 11.91 (s, 1H, NH); ${ }^{13} \mathrm{C}$ NMR (125 MHz, $\left.\mathrm{CDCl}_{3}\right) \delta: 13.55,14.39,14.48,24.26$, $24.51,38.77,111.662,126.16,127.87,133.87,134.72$, $134.91,140.72,141.19,150.84,164.15,181.38$; HRMS calcd for $\mathrm{C}_{17} \mathrm{H}_{23} \mathrm{~N}_{4} \mathrm{OS}[\mathrm{M}+\mathrm{H}]^{+}$331.1587, found 331.1588 .

\section{2 .2 生物活性测试}

试验对象: 番茄早疫病菌(Alternaria solani)、小麦赤 霉病菌 (Fusahum graminearum)、马铃薯晚疫病菌 (Phytophthora infestans)、辣椒疫霉病菌(Phytophthora capsici)、黄瓜灰霉病菌(Botrytis cinerea)、油菜菌核病菌 (Sclerotinia sclerotiorum)、水稻纹枯病菌(Thanatephorus cucumeris)、黄瓜枯萎病菌(Fusarium oxysporum)、花生 褐斑病菌 (Cercospora arachidicola)、苹果轮纹病菌 (Botryospuaeria berengeriana).

试验处理：化合物 $\mathbf{1}$ 用 DMF 溶解成 $1 \% \mathrm{EC}$ 备用. 采 用菌丝生长抑制法，评价供试化合物在 $50 \mu \mathrm{g} / \mathrm{mL}$ 剂量 下对 10 种试验靶标的室内杀菌活性, 活性测试结果如 表 1 所示.

辅助材料(Supporting Information) 化合物 $6 \mathrm{a} \sim 6 \mathrm{k}$ 的 ${ }^{1} \mathrm{H}$ NMR 和 HRMS 图谱. 这些材料可以免费从本刊网站 (http://sioc-journal.cn/)上下载.

\section{References}

[1] Russell, P E. Outlooks Pest Manage. 2009, 20, 122

[2] Mu, J. X.; Shi, Y. X.; Yang, M. Y.; Sun, Z. H.; Liu, X. H.; Li, B. J.; Sun, N. B. Molecules 2016, 21, 68.

[3] Elbe, H.-L.; Rieck, H.; Dunkel, R.; Wachendorff-Neumann, U.; Mauler-Machnik, A.; Kuck, K.-H.; Kugler, M.; Jaetsch, T. WO 2002008195, 2002 [Chem. Abstr. 2002, 136, 151162].

[4] Elbe, H.-L.; Rieck, H.; Dunkel, R.; Zhu-Ohlbach, Q.; MaulerMachnik, A.; Wachendorff-Neumann, U.; Kuck, K.-H. WO 2003010149, 2003 [Chem. Abstr. 2003, 136, 137308].

[5] Du, S.; Tian, Z.; Yang, D.; Li, X.; Li, H.; Jia, C.; Che, C.; Wang, M.; Qin, Z. Molecules 2015, 20, 8395.

[6] Xiong, L.; Zhu, X. L.; Shen, Y. Q.; Wishwajith, W. K. W. M.; Li, K.; Yang, G. F. Eur. J. Med. Chem. 2015, 95, 424.

[7] Xiong, L.; Zhu, X. L.; Gao, H. W.; Fu, Y.; Hu, S. Q.; Jiang, L. N.; Yang, W. C; Yang, G. F. J. Agric. Food Chem. 2015, 64, 4830.

[8] Liu, X. H.; Zhao, W.; Shen, Z. H.; Xing, J. H.; Yuan, J.; Yang, G.; Xu, T. M.; Peng, W. L. Bioorg. Med. Chem. Lett. 2016, 26, 3626.

[9] Zhao, W.; Xing, J. H.; Xu, T. M.; Peng, W. L.; Liu, X. H. Front. Chem. Sci. Eng. 2017, 11, 363.

[10] Zhao, W.; Shen, Z. H.; Xu, T. M.; Peng, W. L.; Liu, X. H. Lett. Drug Des. Discovery 2017, 14, 323.

[11] Zhao, W.; Shen, Z. H.; Xing, J. H.; Yang, G.; Xu, T. M.; Peng, W. L.; Liu, X. H. Chem. Pap. 2017, 71, 921.

[12] Zhao, W.; Shen, Z. H.; Xing, J. H.; Xu, T. M.; Peng, W. L.; Liu, X. H. Chin. J. Struct. Chem. 2017, 36, 423.

[13] Liu, X. H.; Tan, C. X.; Weng, J. Q. Phosphorus, Sulfur Silicon Relat. Elem. 2011, 186, 558.

[14] Zhai, Z. W.; Wang, Q.; Shen, Z. H.; Tan, C. X.; Weng, J. Q.; Liu, X. H. Chin. J. Org. Chem. 2017, 37, 232 (in Chinese) (翟志文, 汪乔, 沈钟华, 谭成侠, 翁建全, 刘幸海, 有机化学, 2017, 37, 232.) 
[15] Chen, W.; Wei, W.; Wu, C. C.; Li, Y. X.; Li, Y. H.; Yu, S. J.; Li, Z. M. Chin. J. Org. Chem. 2015, 35, 1576 (in Chinese).

(陈伟, 魏巍, 吴长春, 李玉新, 李永红, 于淑晶, 李正名, 有机 化学, 2015, 35, 1576.)

[16] Chen, Y. W.; Wan, Y. Y.; Liu, Q. X.; Liu, J. B.; Xiong, L. X.; Yu, S. J.; Li, Z. M. Chin. J. Org. Chem. 2015, 35, 882 (in Chinese) (陈有为, 万莹莹, 刘巧霞, 刘敬波, 熊丽霞, 于淑晶, 李正名, 有机化学, 2015, 35, 882.)

[17] Li, C. K.; Jiang, L.; Wang, Y.; Wan, F. X.; Zhang, P. Z.; Li, Y.; Cui, Z. N. Chin. J. Org. Chem. 2014, 34, 2296 (in Chinese) (李成坤, 姜林, 王悦, 万福贤, 张沛之, 李映, 崔紫宁, 有机化 学, 2014, 34, 2296.)

[18] Liu, X. H.; Wang, Q.; Sun, Z. H.; Wedge, D. E.; Becnel, J. J.; Estep, A. S.; Tan, C. X.; Weng, J. Q. Pest Manage. Sci. 2017, 73, 953.

[19] Chen, J. N.; Wang, X. F.; Li, T.; Wu, D. W.; Fu, X. B.; Zhang, G. J.; Shen, X. C.; Wang, H. S. Eur. J. Med. Chem. 2016, 107, 12.

[20] Liu, X. H.; Zhai, Z. W.; Xu, X. Y.; Yang, M. Y.; Sun, Z. H.; Weng, J. Q.; Tan, C. X.; Chen, J. Bioorg. Med. Chem. Lett. 2015, 25, 5524

[21] Zhai, Z. W.; Shi, Y. X.; Yang, M. Y.; Zhao, W.; Sun, Z. H.; Weng, J.
Q.; Tan, C. X.; Liu, X. H.; Li, B. J.; Zhang, Y. G. Lett. Drug Des. Discovery 2016, 13, 521

[22] Liu, X. H.; Tan, C. X.; Weng, J. Q. Phosphorus, Sulfur Silicon Relat. Elem. 2011, 186, 552.

[23] Zhang, L. J.; Yang, M. Y.; Sun, Z. H.; Tan, C. X.; Weng, J. Q.; Wu, H. K.; Liu, X. H. Lett. Drug Des. Discovery 2014, 11, 1107.

[24] Liu, X. H.; Fang, Y. M.; Xie, F.; Zhang, R. R.; Shen, Z. H.; Tan, C. X.; Weng, J. Q.; Xu, T. M.; Huang, H. Y. Pest Manage. Sci. 2017, $73,1900$.

[25] Yan, S. L.; Yang, M. Y.; Sun, Z. H.; Min, L. J.; Tan, C. X.; Weng, J. Q.; Wu, H. K.; Liu, X. H. Lett. Drug Des. Discovery 2014, 11, 940.

[26] Sun, N. B.; Shen, Z. H.; Zhai, Z. W.; Wu, H. K.; Weng, J. Q.; Tan, C. X.; Liu, X. H. Chin. J. Org. Chem. 2017, 37, 2044 (in Chinese). (孙娜波，沈钟华，翟志文，武宏科，翁建全，谭成侠，刘幸海, 有机化学, 2017, 37, 2044.)

[27] Zhao, W.; Shen, Z. H.; Xu, T. M.; Peng, W. L.; Liu, X.-H. J. Heterocycl. Chem. 2017, 54, 1751.

[28] Liu, X. H.; Zhao, W.; Shen, Z. H.; Xing, J. H.; Xu, T. M.; Peng, W. L. Eur. J. Med. Chem. 2017, 125, 881.

(Li, L.; Fan, Y.) 